\title{
Metformin extends the lifespan of iMSUD mice by rescuing physiologic dysfunction and modulating mechanisms of mTORC1 activation
}

\section{Yuehmei Hsu}

Buck Institute for Research on Aging

\section{Sonnet Davis}

Buck Institute for Research on Aging

Chen-Yu Liao

Buck Institute for Research on Aging

\section{Samuel Larson}

Buck Institute for Research on Aging

\section{Vanessa Nava}

Buck Institute for Research on Aging

\section{Junying Wang}

Buck Institute for Research on Aging

\section{Delana Miller}

Buck Institute for Research on Aging

\section{Brian Kennedy}

National University Singapore Yong Loo Lin School of Medicine

Arvind Ramanathan ( $\nabla$ arvind@instem.res.in )

Institute for Stem Cell Biology and Regenerative Medicine https://orcid.org/0000-0002-0375-3740

\section{Research}

Keywords: Branched chain amino acids, Maple Syrup Urine Disease, metformin, mTORC

Posted Date: July 8th, 2020

DOI: https://doi.org/10.21203/rs.3.rs-39327/v1

License: () (i) This work is licensed under a Creative Commons Attribution 4.0 International License. Read Full License 


\section{Abstract}

Background-Dysregulation of branched chain amino acid (BCAA) catabolism caused by mutations in the metabolizing enzyme-branched chain alpha-keto acid dehydrogenase (BCKDH) leads to the fatal newborn disorder Maple Syrup Urine Disease (MSUD) and is linked to chronic diseases such as type 2 diabetes.

Results- Here we show that a MSUD mouse model with severely reduced BCKDH activity exhibits glucose intolerance, altered mTOR signaling, decreased levels of TCA cycle intermediates, reduced distribution of Type I skeletal muscle fibers and activated AMP-activated protein kinase (AMPK) signaling, all of which can be ameliorated by long term treatment with metformin, which enhances the survival of these mice. Treatment with metformin was correlated with increased serum-levels of growth/differentiation factor GDF15 (GFD15) and adiponectin. We also found that branched chain keto acid regulates leucinemediated activation of mTORC1 by preventing the dissociation of sestrin2-gator complex in vitro.

Conclusions-In summary, our study suggests metformin could be an effective therapeutic candidate for MSUD patients and maps the crosstalk between key pathways that maintain metabolic homeostasis in MSUD.

\section{Introduction}

Branched chain amino acids (BCAA, including valine, isoleucine and leucine) are essential amino acids that directly activate the mammalian target of rapamycin (mTOR) nutrient signaling pathway $(1-3)$ and play an important role in skeletal muscle metabolism, homeostasis and exercise-adaptation $(4,5)$. More directly, mutations in the gene for BCAA metabolizing enzyme- branched chain alpha-ketoacid dehydrogenase (BCKDH) cause the rare, debilitating Maple Syrup Urine Disease (MSUD) (6-8). Dysfunction in BCAA metabolism and an increase in fasting concentrations of BCAA are also associated with insulin resistance and type 2 diabetes in both animal models and human patients $(9,10)$. Despite this accumulating evidence, it is unclear whether enzymes that control the metabolism of BCAA play a direct role in controlling glucose metabolism and physiology. Insights from previous studies (11) show that over half the capacity to metabolize BCAAs resides in skeletal muscle, with significant activity also in adipose tissue, liver and brain. The metabolism of these BCAAs (leucine, isoleucine and valine) occurs through two common enzymes, BCAA amino transferase (BCAT1 and 2) and BCKDH. BCATs use mitochondrial alpha-ketoglutarate as a substrate for the amino-transferase reaction, producing ketoisovaleric (KIV), keto-methylvaleric (KMV) and keto-isocaproic (KIC) acids from Valine, Isoleucine and leucine, respectively. The next enzyme in the pathway, $B C K D H$, represents the first irreversible 'flux generating' step. In the classic form of $\operatorname{MSUD}(6,7)$, the BCKDH enzyme exhibits only $2-5 \%$ of its normal activity. In addition to the classic form, there are intermediate (12) (15-25\% BCKDH activity) and intermittent (asymptomatic until 10-16 months or later) forms. 
It has been recognized that MSUD patients display skeletal-muscle abnormalities, including lesions in myofibers (13). The mechanistic basis for skeletal muscle abnormalities in MSUD is not understood, and targeting this dysfunction has the potential to have important therapeutic outcomes in MSUD patients. Animal models are well suited to explore the pathophysiology of MSUD and permit pre-clinical testing of drug interventions. Here we used the intermediate MSUD (iMSUD) model generated by Homniacs et. al. (7) which develops pathology similar to MSUD patients and survives past weaning. In our previous study, using a combination of metabolic profiling and small molecule screening, we found that an acute treatment with metformin, a widely used anti-diabetic drug, diminishes MSUD-associated ketoacidosis in patient-derived fibroblasts and in an iMSUD mouse model (14). Here, we studied the long-term beneficial effects of metformin in iMSUD mice. Our studies reveal that BCAA metabolism plays a key role in maintaining TCA metabolite levels, glucose homeostasis and skeletal muscle function. These aspects are impacted by metformin in iMSUD mice. Finally, our studies reveal a novel arm of mTOR regulation by the leucine metabolite KIC and KMV, whose accumulation can prevent the canonical leucine-mediated activation of mTOR.

\section{Results}

\section{Metformin induces lifespan-extension and body weight- maintenance in iMSUD mice}

Our previous study has identified that metformin inhibits BCAA-derived ketoacidosis and promotes metabolic homeostasis in MSUD patient-derived fibroblast cells (14). To determine whether metformin extends the survival and/or delays pathology in iMSUD mice, we used the strategy of delivery in the food as described previously (15). We found metformin did not measurably alter the body weight and median survival of iMSUD mice (Figure S1A and B), which live approximately two month of age. We note, however, one observation about the metformin survival curve that merits discussion. One mouse treated with metformin survived to 120 days, which is longer than we have ever observed with these mice. This divergence at the end of the survival curve, while not statically significant, warrants consideration in design of different delivery strategy of metformin. In addition, iMSUD is an inborn error of metabolism in both human patient and mouse disease model $(6-8,16)$. Considering that the chow-mediated administration of metformin could occur only after weaning (21 days of age) and the dosage of metformin can be variable based on food consumption, we next treated iMSUD mice with different doses of metformin by daily intra-peritoneal (IP) injection started at 5 days of age. Low-dose $(125 \mathrm{mg} / \mathrm{kg} \mathrm{bw})$ administration of metformin increased the survival of iMSUD mice in both sexes (Fig. 1A and B). The survival is dose-dependently increased at a higher dose $(250 \mathrm{mg} / \mathrm{kg} \mathrm{bw})$ of metformin with a median survival of 32 days (metformin-treated) vs 24 days (PBS-treated) in females and a median survival of 33 days (metformin-treated) vs 24 days (PBS-treated) males (Fig. 1A and 1B, Log-rank (Mantel-Cox) test, $\mathrm{P}<$ 0.05). In our study, iMSUD mice weigh about half as much as wild type mice at weaning (Figure S1B). We found only the higher dosage of metformin $(250 \mathrm{mg} / \mathrm{kg} \mathrm{bw})$ caused a continuous increase in body weight of iMSUD mice over their lifespan (Fig. 1C and D). 
Metformin has been shown to mediate body weight and energy balance through increasing the circulating levels of the peptide hormone growth/differentiation factor 15 (GDF15) (17). GDF15 has also been shown to lower body weight through a brain-stem-restricted receptor (18). In order to investigate the effect of metformin on the increase in body weight of iMSUD mice, we measured the concentration of GDF15 in serum of metformin-treated iMSUD mice. We found the concentration of GDF15 was increased after metformin treatment in both wild type (WT) and iMSUD mice (Fig. 1E). We also measured the concentration of adiponectin - another adipokine regulates body weight. We found that the concentration of adiponectin in the serum was also increased upon metformin treatment (Fig. 1F). This suggests that the increased GDF15 concentration is dependent on the treatment of metformin in both wild type and iMSUD mice. However, the increase in total fat content was only observed in metformin-treated iMSUD mice (Fig. 1G). To further support of this finding, we measured the protein-expression level of fatty acid synthesizing enzyme fatty acid synthetase (FAS) in metformin-treated iMSUD mice. We found that FAS protein expression was increased by metformin in both skeletal muscle and subcutaneous fat (ingWAT) of iMSUD mice (Fig. 1H). These results suggest that metformin-induced body weight increase might depend on the increase in fatty acid synthesis pathway in both adipose tissue and skeletal muscle in iMSUD mice.

\section{Metformin-induced decreased glucose intolerance in iMSUD mice}

Previous studies suggest that elevated circulating BCAA levels have been correlated with severity of insulin resistance $(19,20)$. Metformin has been known to improve the regulation of glucose tolerance (21-23). Therefore, we tested the physiological effects of BCKDH loss on the glucose homeostasis of iMSUD mice. We found there was significant glucose intolerance in iMSUD mice (Fig. 2A and B). The increased area under the curve (AUC) demonstrates that iMSUD mice have dysregulated glucose disposal capacity. We also found that metformin restored glucose tolerance in iMSUD mice to levels similar to that of wild type mice (Fig. 2A). This result of BCAA metabolism via BCKDH controls glucose homeostasis suggests a mechanistic role and not merely a correlative role for BCAA metabolism in controlling glucose homeostasis. To test this hypothesis, we then investigated whether the iMSUD-related dysfunction in glucose tolerance and its rescue by metformin were related to the levels of insulin and fasting glucose. We found that there was a decrease in insulin level in the serum of iMSUD mice (Fig. 2C), but this was not rescued by the treatment with metformin. There was a decrease in fasting glucose levels in iMSUD mice, which was also not significantly affected by treatment with metformin (Fig. 2D). These results suggest that metformin-induced decreased glucose intolerance in iMSUD mice is not involved with modulation of insulin and glucose level.

It is well known that metformin regulates the protein activation/expression of IGF-1 receptor, which plays an important role in regulating glucose uptake into skeletal muscle and liver (24). Therefore, we measured IGF-1 signaling in metformin-treated liver and skeletal muscle samples using western blotting. We found that the expression/activation of IGF-1R was increased in the liver of metformin-treated iMSUD mice, 
which is consistent with previous reports (Figure S2A) (25). The expression/activation of IGF-1R was diminished in the skeletal muscle of iMSUD mice, and metformin mitigated this effect (Figure S2A).

\section{Metformin rescues muscle dysfunction in iMSUD mice}

It has been recognized that MSUD patients display skeletal-muscle abnormalities, including lesions in myofibers (13). Our previous study shows reduced mean fiber cross-sectional area in quadriceps and gastrocnemius muscles of iMSUD mice (14). The result of lifespan extension by metformin treatment suggests the skeletal muscles of iMSUD mice might be the target of metformin treatment. Force measurement revealed that iMSUD mice have lower grip strength when compared to wild type mice (Fig. 3A), demonstrating a muscle functional deficit in iMSUD mice. We next analyzed the effect of BCKDH dysfunction on skeletal muscle fiber type distribution. Gastrocnemius and soleus skeletal muscle sections were stained for muscle fiber-type I (slow twitch oxidative), IIA (fast twitch oxidative) and IIB (fast twitch glycolytic) using previously characterized antibodies (26) (Fig. 3B). Both muscle groups showed similar trends, but the most significant effects were observed in soleus, where iMSUD mice have decreased levels of the slow twitch oxidative fiber-type I and increased levels of fast twitch glycolytic fiber-type IIB (Fig. 3C). No changes were observed in type IIA fiber content. Type I fibers are associated with higher mitochondrial metabolism, and their loss has been shown to be correlated with metabolic diseases such as Type II diabetes $(27,28)$. Treatment of iMSUD mice with metformin restored the levels of type I fibers in iMSUD mice nearly to that of wild type mice (Fig. 3C). Metformin partially restored the grip strength of iMSUD mice (Fig. 3A), showing that the rescue in fiber type composition was correlated with improvement in skeletal muscle function.

\section{Metformin reverses elevated AMPK signaling in iMSUD mice}

It's well known that metformin activates AMPK, including hepatocytes (29) and muscle cells (22, 30-32). We found that iMSUD mice had increased levels of phosphorylated AMPK (Thr172) and the canonical AMPK target, ACC (Ser79) in skeletal muscle or liver tissues (Fig. 3D), which suggests that loss of BCKDH activates the AMPK energy-stress response pathway. The treatment of metformin had increased phosphorylation of both AMPK and ACC in wild type mice. Surprisingly, metformin has an opposite effect in iMSUD mice and relieves the hyper-activation of the AMPK energy stress pathway (Fig. 3D).

\section{Global dysfunction in oxidative carbon metabolism caused by BCKDH knockdown}

To gain a broader picture of physiological dysfunction in iMSUD mice, we performed metabolic profiling of tissue and serum. We profiled relative levels of BCAA, leucine derived KIC and TCA metabolites from liver, skeletal muscle and serum (Fig. 4A, Figure S3 and S4). As expected, we found an accumulation of leucine/isoleucine and keto-isocaproic acid in all tissues and serum. Treatment with metformin was able to diminish the accumulation of leucine/isoleucine and KIC significantly in liver, skeletal muscle tissue and serum. Interestingly, iMSUD mice had significantly lower overall metabolism as judged by a decrease 
in TCA cycle metabolites and lactate in tissue and serum. The final break-down product of BCAA (including leucine) is the mitochondrial anaplerotic substrate acetoacetate. Acetoacetate is readily converted to beta-hydroxybutyrate $(\beta \mathrm{HB})$ by $\beta \mathrm{HB}$ dehydrogenase. The levels of $\beta \mathrm{HB}$ were significantly decreased in skeletal muscle of iMSUD mice (Fig. 4A, Figure S4). Metformin has been shown to promote mitochondrial metabolism via regulation of redox state (33-37), activation of AMPK (38-42) and increased the production of lactate (43-46). Here, metformin was unable to restore intra-tissue lactate or TCA metabolite levels in iMSUD mice unlike in wild type mice, suggesting that BCKDH function is required for metformin action (Fig. 4A). To test whether the role of BCKDH in TCA and glucose metabolism observed in vivo was cell autonomous, we studied the effects of knocking down BCKDH using cultured cell system (Fig. 4B). Upon BCKDH knock down using shRNA in mouse C2C12 myoblasts, we observed an extracellular accumulation of KIC and KMV as expected. Intriguingly, there was a dramatic decrease in TCA and glycolytic metabolites in C2C12 shBCKDH cells, confirming that BCKDH plays a critical role in regulating TCA and glycolytic homeostasis. Finally, treatment with $1 \mathrm{mM}$ metformin was unable to promote glycolytic and TCA metabolism in shBCKDH unlike in shNT (scrambled non-targeting shRNA control) $\mathrm{C} 2 \mathrm{C} 12$ cells. This also confirms that metformin requires functional $\mathrm{BCKDH}$ for its metabolic action.

\section{iMSUD mice have diminished mTOR signaling, which is modulated by metformin}

Global changes in mitochondrial and energy metabolism in iMSUD mice suggest that the mTOR signaling pathway, a key nutrient sensor and controller cellular physiology (3) may be altered. To test our hypothesis, we examined the activation of mTORC1 and mTORC2 in the skeletal muscle tissue of iMSUD mice. Our results indicate that both mTORC1 (P-S6) and mTORC2 (P-Akt) signaling is decreased in iMSUD mice (Fig. 5A). Treatment with metformin increased the activation in both mTORC1 and mTORC2 signal pathways. It is well known that the BCAA leucine is a direct activator of mTORC1 signal pathway (47-49). One of the hallmarks of iMSUD mice is the accumulation level of leucine, which rises up to $0.5 \mathrm{mM}$ and $\mathrm{KIC}$ up to $1 \mathrm{mM}$ in serum (14). It was interesting that metformin-mediated induction of mTOR signaling was correlated with diminished KIC levels (Fig. 5A), possibly suggesting that KIC may play a role in the inhibition of mTOR signaling.

Leucine is known to activate the mTORC1 signaling complex through stabilization of the GATOR1 complex by direct binding to the sestrin-2 protein (50). Activation of mTORC1 signaling complex causes the re-localization of the mTORC1 complex to the lysosomal membrane (51). To investigate the effect of accumulated $\mathrm{KIC}$ or KMV on mTORC1 complex activation, we tested whether KIC and/or KMV could directly modulate mTORC1 activity in HEK293T cells. Both mTORC1 and LAMP1 proteins (a lysosomal marker) were immuno-stained, and cells were imaged to assess the co-localization of mTOR and the lysosomal compartment as a readout of mTORC1 activation. Interestingly, treatment with $1 \mathrm{mM} \mathrm{KIC}$ and/or $1 \mathrm{mM} \mathrm{KMV}$ inhibited leucine-induced mTORC1 translocation (Fig. 5B). 
To further examine the downstream regulating mechanisms of mTORC1 activation by accumulated KIC or KMV, we tested the effect of KIC and KMV on leucine-induced phosphorylation of the canonical mTOR substrate p70S6K (Fig. 5C), finding that treatment with KIC or KMV interfered with p70S6K phosphorylation. In HEK293T cells, the activation of mTORC1 can be induced by both leucine and arginine $(3,47,51)$. We then tested whether this effect was specific to leucine-induced mTORC1 activation by alternatively activating $\mathrm{mTORC} 1$ phosphorylation using arginine, which disrupt the CASTOR1-GATOR2 protein complex by binding to CASTOR1 and then activate mTORC1 in cells (52). Interestingly, KIC and KMV co-treatment had no effect on arginine-mediated activation of mTORC1 (Fig. 5C).

It has been shown that leucine induces the disruption of sestrin2-GATOR2 interaction to regulate mTORC1 activation (47). To investigate whether KIC could affect the leucine-induced disruption of sestrin2-GATOR2 interaction, HEK293T cells overexpressing FLAG-sestrin2 were treated with leucine and KIC or KMV. We immunoprecipitated FLAG-sestrin2 and probed for pulldown of two GATOR2 complex components, WDR59 and MIOS. We found that leucine treatment disrupted the interaction of sestrin2 with both MIOS and WDR59 as expected. Interestingly, both KIC and KMV promoted the interaction of sestrin2 with MIOS and WDR59, even upon leucine co-treatment (Fig. 5D). These results suggest that pathogenic accumulation of KIC or KMV in iMSUD mice could block leucine-induced activation of mTORC1 by preventing the dissociation of the sestrin2-GATOR2 complex (Fig. 5E).

\section{Discussion}

The findings in this study support the idea that metformin may be an effective therapeutic agent for MSUD. Metformin enhances survival in both sexes and promotes weight maintenance (Fig. 1A-D). In this context, metformin increases the fat content of iMSUD mice and concomitantly increases the expression of both the fatty acid synthase (FAS) enzyme and the adipokine adiponectin (Fig. 1E). Recently it has been shown that the levels of GDF15 are increased upon metformin treatment, and that this hormone modulates the effects of metformin on weight loss upon high-fat diet condition (53). It is interesting that treatment of both iMSUD and wild type mice show an increase in levels of GDF15 in the serum, but this instead results in gain in weight and fat content in iMSUD mice. In our study, metformin induces the body weight loss in wild type mice (Figure S1B) which is consistent with previous finding. These results suggest that the effect of GDF15 might depend on the genetic content and metabolic background of mice. The adipokine adiponectin has been shown to improve glucose metabolism, lipid metabolism and insulin sensitivity in numerous tissues (54-56) and therefore it is interesting that its levels are increased with metformin treatment. The resting insulin levels do not change upon metformin treatment (Fig. 2C), therefore it is possible that the effects of adiponectin might be via improvement of insulin signaling and glucose homeostasis. iMSUD mice have decreased glucose tolerance (Fig. 2A and B), lower insulin levels (Fig. 2C) and decreased fasting glucose levels (Fig. 2D). Therefore, loss of BCKDH activity associated with iMSUD causes altered glucose homeostasis. It is known that perturbations in BCAA levels in the serum are closely associated with loss of glucose homeostasis and risk of Type II Diabetes (57-60). The iMSUD mice show that a functional BCKDH is required to maintain glucose homeostasis. Treatment with 
metformin increased IGF-R phosphorylation associated with improved insulin signaling (Figure S2) and restored glucose clearance capacity (Fig. 2B), without increasing levels of insulin in the serum (Fig. 2C) or improving fasting glucose (Fig. 2D). This suggests that metformin is not able to restore all aspects of glucose homeostasis, but possibly improves insulin signaling and glucose disposal in iMSUD mice.

Study shows that skeletal muscle tissue is a major site of glucose disposal upon metformin treatment $(22,61)$. Our previously result shows that in iMSUD mice there is a loss of skeletal muscle mass (14). More specifically, we show here that it is the loss of the mitochondrial-rich Type I fibers (Fig. 3B and C) in iMSUD mice, which is partially restored by metformin treatment. This also corresponds to the increased grip strength in mice treated with metformin, which shows a functional improvement of this tissue.

The iMSUD related loss of BCKDH causes decreased TCA components both in vitro and in vivo (Fig. 4A and $B$ ), suggesting that BCAA are a significant source of TCA intermediates in all tissues tested, but most significantly in liver. This decrease in TCA intermediates is correlated with the diminished energy supply, as judged by the activation of the AMPK pathway (Fig. 3D). It has been proposed that metformin activates AMPK $(38,40,42)$ and in agreement with this, wild type mice have increased phosphorylation of both AMPK and ACC upon metformin treatment. Surprisingly, metformin has the opposite effect in iMSUD mice (Fig. 4D). These data suggest that the previously characterized activation of AMPK by metformin is mostly observed in the wild type mice $(22,30-32)$, and that the idea of metformin as an AMPK activator does not extend to all metabolic contexts, especially when the activity of BCKDH is reduced. Future studies are needed to assess the effects of metformin in other altered metabolism contexts.

The activity of the mTOR pathway is lower or unchanged in iMSUD in skeletal muscle tissue of iMSUD mice. This is striking since these mice have significantly high levels of leucine the canonical activator of mTOR (47). We present evidence that suggests the inhibitory effects of the metabolites KIC and KMV on mTOR signaling using HEK293T cells imaging of mTOR translocation to lysosomes (Fig. 5B) and western blotting on p70S6K phosphorylation (Fig. 5C). Our results suggest that iMSUD related accumulation of metabolites KIC and KMV might inhibit the leucine mediated activation of mTOR signaling. Our work also suggests that one of the ways by which these organic acids might affect mTOR is by preventing the leucine mediated dissociation of the sestrin2-GATOR2 association (Fig. 5D and E). Understanding the connectivity between BCAA catabolism and mTOR signaling is critical because both pathways are important for proper metabolism and are altered both by aging and in the context of metabolic disease.

\section{Conclusion}

Overall, we show that the iMSUD associated loss of BCKDH controls TCA and glucose homeostasis, and that its dysfunction results in the accumulation of KIC and KMV, which can inhibit the leucine-induced activation of mTOR. Therefore, mTOR positively senses leucine levels, but is also sensitive to the status of BCAA metabolism via KIC or KMV. The mechanism of action of metformin depends on the metabolic 
background, and it acts as an AMPK activator in iMSUD instead of its canonical role as an AMPK inhibitor.

\section{Materials And Methods}

\section{Mouse strain, housing, diets and drug treatments}

The iMSUD and wild-type mice used in this study were obtained by breeding $\mathrm{Dbt}^{\mathrm{tm} 1 \mathrm{Geh} /+} ; \mathrm{Tg}$ (tetODBT)A1 Geh/J; $\mathrm{Tg}$ (Cebpb-tTA)5Bjd) mice to generate iMSUD or Dbt1 ${ }^{+/+}$(wild type) mice homozygous for the LAP-tTA and TRE-E2 transgenes (6). All the mice have free access to water and food and all animal care and experimental procedures were approved by the Institutional Animal Care and Use Committee at the BUCK Institute for Research on Aging. For the lifespan study with metformin diet, the wild type and iMSUD mice were supplied with control (5LG6, TestDiet) or metformin (5LG6 w/0.1\% metformin, TestDiet) after weaning. The mice were regrouped after genotyping and assigned group of treatments were continued till naturally death happened.

For the intraperitoneal injection of metformin of the lifespan study in iMSUD mice, PBS-dissolved metformin solution was given to mice at 125 or $250 \mathrm{mg} / \mathrm{kg}$ of mouse body weight via intraperitoneal injection daily started from 5-days-old age. After determination of genotype after weaning, both wild type and iMSUD mice were continually given injected till natural deathbed occurred.

\section{Mouse physiological measurements}

Body fat composition was determined by in vivo magnetic resonance tomography imaging (EchoMRI) at 3 weeks-old age. The muscle strength was determined by a grip strength behavior task. The grasp strength of each mouse of fore limbs was measured on a pull-grid assembly connected to a grip strength meter (Bioseb). The mouse was drawn along a straight-line parallel to the grid until the grip was broken, providing the peak force in grams. This was repeated at least 4 times within 5 mins intervals between measurements.

Glucose tolerance tests were performed at 4 weeks-old mice. Overnight fasted (16 hrs) mice with free access to drinking water were received $2 \mathrm{~g} / \mathrm{kg}$ body weight of glucose intraperitoneally, and blood glucose levels were measured before and 30,60, 90, and 120 min after glucose injection.

\section{Insulin, adiponectin and GDF15 measurement}

The determination of concentration of insulin (Catalog\# 90080, Crystal Chem), adiponectin (Catlog\# MRP300, R\&D System) and GDF15 (Catlog\# MGD150, R\&D System) in serum is performed under the instruction provided by the company. 


\section{Mouse tissue collection, preparation and immunoblot analysis}

At age of 4-5 weeks, mice were euthanized, and various tissue samples were immediately harvested, snap-frozen by liquid nitrogen and stored at -80 freezer. Total protein was obtained from liver, muscle, and adipose (ingWAT) tissues, quantified by BCA assay (Pierce Biotechnology, Rockford, IL) and SDS-PAGE separated. Antibodies rabbit anti-mouse for P-IGF-1R, P-Akt ${ }_{S 473}, \mathrm{P}_{-S 6} 6_{\mathrm{S} 32}, \mathrm{P}-\mathrm{P} 70 \mathrm{~S} 6 \mathrm{~K}_{\mathrm{T} 172}$, total FAS, Akt, S6, IGF-1R, P70S6K, sestrin2, WDR59, MIOS and GAPDH (Cell Signaling Technology Inc., Danvers, MA) were used and bands were quantified by ImageJ 1.46 r.

\section{Immunofluorescence staining}

For the muscle fiber type determination, skeletal muscle samples of mice were incubated in $0.5 \%$ paraformaldehyde for 3-6 hrs and then incubated with $30 \%$ sucrose/PBS at $4^{\circ} \mathrm{C}$ overnight. The skeletal muscle samples are embedded using OCT (Tissue-Tek) and then cut into 10 um thick cross-sections with a cryostat. Immunofluorescence analysis of $\mathrm{MHC}$ expression was performed with primary antibodies against MHC1 (BA-F8, DSHB), MHClla (SC-71, DSHB) and MHCllb (BF-F3, DSHB). Slides were visualized using confocal microscope (Nikon) and final panaroma images were composed using Image composer editor software (Microsoft research).

\section{Cell line}

Human HEK293T cells (CRL-3216) and mouse C2C12 cells (CRL-1722) were purchased from ATCC (ATCC.org). Cells were grown at $37^{\prime} \mathrm{C}$ and $5 \%$ carbon-di-oxide. Cells were grown in standard tissue culture treated dishes purchased from Corning. Inc. Cells were grown in standard DMEM media (Corning) with $5 \mathrm{mM}$ glucose and 10\% FBS (Corning Catalog \# 35-010-CV). Cells were seeded at approximately 50\% confluency or at 5000 cells per 96 -well plate and 50,000 cells per 6 -well plate. Media was changed every 72 hours. Cells were split at $1: 5$ ratio upon reaching $80 \%$ confluency. Differentiation of C2C12 cells was initiated by $10 \%$ FBS serum withdrawal and changed into $2 \%$ hourse serum in growth media. PenicillinStreptomycin was added during passaging.

\section{Immunoprecipitation assay}

After HEK293T cells were treated with indicated stimulation for $10 \mathrm{~min}$, cells were rinsed one time with ice-cold PBS and immediately lysed with Triton lysis buffer (1\% Triton, $10 \mathrm{mM} \beta$-glycerol phosphate, 10 $\mathrm{mM}$ pyrophosphate, $40 \mathrm{mM}$ HEPES pH 7.4, $2.5 \mathrm{mM} \mathrm{MgCl} 2$ and 1 tablet of EDTA-free protease inhibitor (Roche). The cell lysates were cleared by centrifugation at $13,000 \mathrm{rpm}$ at $4^{\circ} \mathrm{C}$ in a microcentrifuge for 10 minutes. Protein concentration was determined by DC assay and $2 \mathrm{mg}$ protein/sample was used for antiFLAG-immunoprecipitations. The FLAG-M2 affinity gel was washed 3 times with lysis buffer. $30 \mu$ of a 
$50 / 50$ slurry of the affinity gel was then added to clarified cell lysates and incubated with rotation for 2 hours at $4^{\circ} \mathrm{C}$. The anti-FLAG-M2 beads were washed two time with lysis buffer. Immunoprecipitated proteins were denatured by the addition of $50 \mu \mathrm{l}$ of sample buffer and boiling for 5 minutes as described, resolved by $8 \%$ SDS-PAGE, and analyzed by immunoblotting.

\section{Viral preparation and transduction}

HEK293T cells were grown in 6-well tissue culture treated dishes to 70-80 \% confluence. Tubes with 125 $\mathrm{ml} 2.5 \mathrm{mM} \mathrm{CaCl}_{2}$ and $375 \mathrm{uL}$ DEPC water were prepared per viral transduction replicate. $500 \mu \mathrm{l}$ of $\mathrm{CaCl} 2$ solution was aliquoted per viral tube. For each virus, $6.5 \mu \mathrm{g}$ of psPAX2 (helper plasmid) and $3.5 \mu \mathrm{g}$ of VSVG plasmid to were added to the master mix tube. $10 \mu \mathrm{g}$ of shRNA plasmid was added with $500 \mu \mathrm{l}$ of $2 \mathrm{X}$ BBS ( $\mathrm{pH}=6.95)$ to each viral tube, and vortexed for 5 minutes. The tube was incubated for 20 minutes at room temperature. The viral solution was added drop-wise to HEK cells. After 48 hours, the viral supernatant was collected and centrifuged at $1200 \mathrm{rpm}$ for 3 minutes. The Supernatant was filtered through a 0.44 micron filter and frozen in $1 \mathrm{ml}$ aliquots at $-80^{\circ} \mathrm{C}$.

Cells were transduced at $50-60 \%$ confluence. Two $\mu$ l of polybrene $(1: 1000)$ was added near the bottom of a $15 \mathrm{ml}$ tube. $1 \mathrm{ml}$ of growth media media was then added per $1 \mathrm{ml}$ of viral particle containing supernatant. After 24 hours, viral media was removed and cells were removed with PBS and fresh media was added. After 72 hours a puromycin selection was performed. For $\mathrm{C} 2 \mathrm{C} 12 \mathrm{cells}, 2 \mu \mathrm{g} / \mathrm{ml}$ puromycin solution was used for selection over 6 days.

\section{Tissue metabolite extraction}

Liquid-Liquid extraction (LLE) was performed based on previously reported protocols $(52,62)$ with some modifications. Approximately $25 \mathrm{mg}$ of cryohomogenized tissue was sonicated on ice with $0.5 \mathrm{ml} 50 \%$ methanol with $2 \mu \mathrm{g} / \mathrm{ml} 13 \mathrm{C} 1$-leucine added as an internal standard. A volume of $1.5 \mathrm{ml} \mathrm{of} \mathrm{CHCl}_{3}$ with $20 \mathrm{pg} / \mathrm{ml}$ heptadecanoic acid was then added to each sample and vortexed for 1 min. Each sample was centrifuged at $10,000 \times \mathrm{g}$ for $30 \mathrm{~min}$ at $4^{\circ} \mathrm{C}$ to precipitate total protein and separate the lipid and aqueous phases. Following centrifugation, $250 \mu \mathrm{l}$ of the aqueous phase and $1.0 \mathrm{ml}$ of the lipid phase were recovered, concentrated by speedvac and $\mathrm{N}_{2}$, respectively, and stored at $-80^{\circ} \mathrm{C}$. The recovered protein fraction was rinsed (3x) with $500 \mu \mathrm{l}$ of methanol, vortexed and centrifuged at $10,000 \mathrm{xg}$ for $10 \mathrm{~min}$. Protein was resuspended in $60 \mu \mathrm{l}$ of $8 \mathrm{M}$ urea $+50 \mathrm{mM}$ TEAB pH 7.5 and sonicated in a water bath for $10 \mathrm{~min}$. Protein was quantified for normalization.

\section{C2C12 intracellular metabolite extraction}

Extracellular (spent) media was removed from $\mathrm{C} 2 \mathrm{C} 12$ cells. C2C12 cells were then rinsed 3 times with phosphate-buffered saline (PBS) and quenched using $1 \mathrm{ml} 50 \%$ methanol. A volume of $2 \mathrm{ml}$ of $\mathrm{CHCl}_{3}$ with 
$20 \mathrm{pg} / \mathrm{ml}$ heptadecanoic acid was added to each sample and mixed for $1 \mathrm{~min}$ at $4^{\circ} \mathrm{C}$. Samples were centrifuged at $4,000 \times \mathrm{g}$ for $30 \mathrm{~min}$ at $4^{\circ} \mathrm{C}$, to fully precipitate total protein and separate aqueous and lipid phases. Following centrifugation, $1.5 \mathrm{ml}$ of aqueous phase were recovered, concentrated by $\mathrm{N}_{2}$, and stored at $-80^{\circ} \mathrm{C}$. The recovered protein fraction was rinsed $(3 \mathrm{x})$ with $500 \mu \mathrm{l}$ of methanol, vortexed and centrifuged at $10,000 \times \mathrm{g}$ for $10 \mathrm{~min}$. Protein was resuspended in $60 \mu \mathrm{l}$ of $8 \mathrm{M}$ urea $+50 \mathrm{mM}$ TEAB pH 7.5 and sonicated in a water bath for $10 \mathrm{~min}$. Protein was quantified for normalization.

\section{C2C12 extracellular aqueous metabolite extraction}

Extracellular (spent) media $(200 \mu \mathrm{l})$ was extracted using $200 \mu \mathrm{l}$ of $100 \%$ methanol with $2 \mu \mathrm{g} / \mathrm{ml}{ }^{13} \mathrm{C} 1$ leucine added as internal standard and $600 \mu \mathrm{l}$ of $\mathrm{CHCl}_{3}$ with $20 \mathrm{pg} / \mathrm{ml}$ heptadecanoic acid and mixed for $1 \mathrm{~min}$ at $4^{\circ} \mathrm{C}$. Samples were centrifuged at $4,000 \times \mathrm{g}$ for $30 \mathrm{~min}$ at $4^{\circ} \mathrm{C}$, to fully precipitate total protein and separate aqueous and lipid phases. Following centrifugation, $100 \mu$ l of aqueous phase was recovered and stored at $-80^{\circ} \mathrm{C}$.

\section{LC-MS analysis}

LC-MS analysis of aqueous metabolites were performed using a UFLC prominence system (Shimadzu, Kyoto, Japan) fitted with following modules: CBM-20A (Communication bus module), DGU-A3 (degasser), two LC-20AD (liquid chromatography, binary pump), SIL-20AC HT (auto sampler) equipped with a Luna (Phenomenex, Torrance, CA, USA) $\mathrm{NH}_{2}(2.0 \mathrm{~mm} \times 150 \mathrm{~mm}, 3.0 \mu \mathrm{M})$ column. A binary gradient was applied. Solvent $A$ consisted of $20 \mathrm{mM}$ ammonium acetate $\mathrm{pH} 9.5$ with $5 \%$ acetonitrile, solvent $B$ was acetonitrile. The starting gradient conditions were $95 \% \mathrm{~B}$ at a flow rate of $0.3 \mathrm{ml} / \mathrm{min}$. The following gradient program was used: 0 to $20 \mathrm{~min}, 95-10 \%$ B, 25-30min $10 \%$ B, and $30.1-35 \mathrm{~min} 95 \%$ B. Samples were kept at $+4^{\circ} \mathrm{C}$, and the injection volume was $10 \mu \mathrm{L}$.

Mass spectrometric analysis was conducted using negative ion electrospray ionization in the multiple reaction monitoring mode (MRM) or multiple ion mode (MI) on an API 4000 QTRAP (Ab Sciex, Foster City, CA, USA) mass spectrometer fitted with a TurboVTM ion source. The ionization parameters were set as follows: curtain gas (CAD); 20psi; collision gas: medium; ion spray voltage (IS): -4500V; Temperature (TEM): $550^{\circ} \mathrm{C}$; Ion source Gas 1 (GS1); $60 \mathrm{psi}$; and Ion source Gas 2 (GS2): 50psi. The compound-specific parameters were established using the appropriate standards. AB SCiex's Analyst ${ }^{\circledR} v 1.6 .1$ was used for all forms of data acquisition and method development.

\section{Quantification and statistical analysis}

AB SCiex's Analyst ${ }^{\circledR V}$ 1.6.1 and Varian Saturn software V6.4 was used for in-depth analysis of the LCMS and GC-MS data, respectively. Software was used for calculating the peak areas for metabolites from muscle and cellular extracts. All other statistical analyses were conducted using GraphPad Prism 8 
(GraphPad, La Jolla, CA, USA). The survival curves were completed using Kaplan-Meier curve. We used a log-rank (Mantel-Cox) test to perform the statistical analyses of the survival curves. All data were presented as mean \pm s.e.m. Comparisons between groups were performed using unpaired, two-tailed Student's t-tests. $P \leq 0.05$ was considered significant and $p \leq 0.005$ considered highly significant.

Quantifications of western blotting were performed using Image J $(\mathrm{NIH})$. Statistical analysis for lifespan studies were performed using Prism 7 v7.01 (GraphPad) software. Data of lifespan study was analyzed utilizing parametric statistics including Student's t test or one-way ANOVA. Data are expressed as mean s.e.m, and statistical significance was accepted when $p<0.05$. Data are expressed as mean s.e.m, and statistical significance was accepted when $p<0.05$.

\section{Data and Software Availability}

The LC-MS data will be available at the NIH Common Fund's Metabolomics Data Repository and Coordinating Center (supported by NIH grant, U01-DK097430) website, the Metabolomics Workbench, http://www.metabolomicsworkbench.org. (The accession numbers for the datasets will be available for access in the near future).

\section{List Of Abbreviations}

iMSUD- intermediate Maple Syrup Urine Disease; BCAA- Branched chain amino acids; KIC- Ketoisocaproic acid. KIV- ketoisovaleric acid; LC-MS- liquid chromatography- mass spectrometry; TCA- tricarboxylic acid

\section{Declarations}

Ethics approval and consent to participate- Not applicable

Consent for publication- Not applicable

Funding- Maple Syrup Urine Disease (MSUD) foundation and NIH R01AG050441-A1.

Author Contributions- Y-M.H., C-Y.L. -experimental design, mouse experiments and biochemical analysis; S.R.L., V.N., J.W. and D.M.M. -mouse works including body weight measurement and genotyping; Y-M.H. in vitro cell culture experiment; S.D. - metabolomics experiment and data analysis; Y-M.H., C-Y.L., B.K.K and A.R. -manuscript preparation.

Acknowledgements- Not applicable

Competing interests- not applicable

Availability of data and materials- The LC-MS data will be available at the NIH Common Fund's Metabolomics Data Repository and Coordinating Center (supported by NIH grant, U01-DK097430) 
website, the Metabolomics Workbench, http://www.metabolomicsworkbench.org. (The accession numbers for the datasets will be available for access in the near future).

\section{References}

1. Arany Z, Neinast M. Branched Chain Amino Acids in Metabolic Disease. Curr Diab Rep. 2018;18:76.

2. Siddik MAB, Shin AC. Recent Progress on Branched-Chain Amino Acids in Obesity, Diabetes, and Beyond. Endocrinol Metab (Seoul). 2019;34:234-46.

3. Saxton RA, Sabatini DM. mTOR Signaling in Growth, Metabolism, and Disease. Cell. 2017;168:96076.

4. Y Hatazawa et al. PGC-1alpha-mediated branched-chain amino acid metabolism in the skeletal muscle. PLoS One. 2014;9:e91006.

5. Shimomura Y, Murakami T, Nakai N, Nagasaki M, Harris RA. Exercise promotes BCAA catabolism: effects of BCAA supplementation on skeletal muscle during exercise. J Nutr. 2004;134:1583S$1587 S$.

6. Skvorak KJ. Animal models of maple syrup urine disease. J Inherit Metab Dis. 2009;32:229-46.

7. Homanics GE, Skvorak K, Ferguson C, Watkins S, Paul HS. Production and characterization of murine models of classic and intermediate maple syrup urine disease. BMC medical genetics. 2006;7:33.

8. Peinemann F, Danner DJ. Maple syrup urine disease 1954 to 1993. J Inherit Metab Dis. 1994;17:315.

9. Lynch CJ, Adams SH. Branched-chain amino acids in metabolic signalling and insulin resistance. Nat Rev Endocrinol. 2014;10:723-36.

10. Newgard CB. Interplay between lipids and branched-chain amino acids in development of insulin resistance. Cell Metab. 2012;15:606-14.

11. Brosnan JT, Brosnan ME. Branched-chain amino acids: enzyme and substrate regulation. J Nutr. 2006;136:207S-211S.

12. Schulman JD, Lustberg TJ, Kennedy JL, Museles M, Seegmiller JE. A new variant of maple syrup urine disease (branched chain ketoaciduria). Clinical and biochemical evaluation. Am J Med. 1970;49:118-24.

13. Ferriere G, de Castro M, Rodriguez J. Abnormalities of muscle fibers in maple syrup urine disease. Acta Neuropathol. 1984;63:249-54.

14. SS D et al. Metformin inhibits Branched Chain Amino Acid (BCAA) derived ketoacidosis and promotes metabolic homeostasis in MSUD. Sci Rep. 2016;6:28775.

15. A Martin-Montalvo et al. Metformin improves healthspan and lifespan in mice. Nat Commun. 2013;4:2192.

16. PR Blackburn et al. Maple syrup urine disease: mechanisms and management. Appl Clin Genet. 2017;10:57-66. 
17. AP Coll et al., GDF15 mediates the effects of metformin on body weight and energy balance. Nature, (2019).

18. S Yatsuga et al. Growth differentiation factor 15 as a useful biomarker for mitochondrial disorders. Ann Neurol. 2015;78:814-23.

19. J Wang et al., BCAA Catabolic Defect Alters Glucose Metabolism in Lean Mice. Frontiers in Physiology 10, (2019).

20. Marshall SM. 60 years of metformin use: a glance at the past and a look to the future. Diabetologia. 2017;60:1561-5.

21. CL Li et al. Effect of metformin on patients with impaired glucose tolerance. Diabet Med. 1999;16:477-81.

22. MP Sajan et al. AICAR and metformin, but not exercise, increase muscle glucose transport through AMPK-, ERK-, and PDK1-dependent activation of atypical PKC. Am J Physiol Endocrinol Metab. 2010;298:E179-92.

23. Sharma S, Wickham E, Nestler J. Changes in Glucose Tolerance with Metformin Treatment in Polycystic Ovary Syndrome: A Retrospective Analysis. Endocr Pract. 2007;13:373-9.

24. AT Alberobello et al. Selective disruption of insulin-like growth factor-1 (IGF-1) signaling via phosphoinositide-dependent kinase-1 prevents the protective effect of IGF-1 on human cancer cell death. J Biol Chem. 2010;285:6563-72.

25. Foretz $M$, Guigas $B$, Viollet $B$. Understanding the glucoregulatory mechanisms of metformin in type 2 diabetes mellitus. Nat Rev Endocrinol. 2019;15:569-89.

26. Kammoun M, Cassar-Malek I, Meunier B, Picard B. A simplified immunohistochemical classification of skeletal muscle fibres in mouse. Eur J Histochem. 2014;58:2254.

27. Stuart CA, et al. Slow-twitch fiber proportion in skeletal muscle correlates with insulin responsiveness. J Clin Endocrinol Metab. 2013;98:2027-36.

28. PH Albers et al. Human muscle fiber type-specific insulin signaling: impact of obesity and type 2 diabetes. Diabetes. 2015;64:485-97.

29. $G$ Zhou et al. Role of AMP-activated protein kinase in mechanism of metformin action. J Clin Investig. 2001;108:1167-74.

30. Bogachus LD, Turcotte LP. Genetic downregulation of AMPK-alpha isoforms uncovers the mechanism by which metformin decreases FA uptake and oxidation in skeletal muscle cells. Am J Physiol Cell Physiol. 2010;299:C1549-61.

31. Fryer LG, Parbu-Patel A, Carling D. The Anti-diabetic drugs rosiglitazone and metformin stimulate AMP-activated protein kinase through distinct signaling pathways. J Biol Chem. 2002;277:2522632.

32. JO Lee et al. Metformin induces Rab4 through AMPK and modulates GLUT4 translocation in skeletal muscle cells. J Cell Physiol. 2011;226:974-81. 
33. Baur JA, Birnbaum MJ. Control of gluconeogenesis by metformin: does redox trump energy charge? Cell Metab. 2014;20:197-9.

34. AR Cameron et al. Metformin selectively targets redox control of complex I energy transduction. Redox Biol. 2018;14:187-97.

35. Kalyanaraman B, et al. Mitochondria-targeted metformins: anti-tumour and redox signalling mechanisms. Interface Focus. 2017;7:20160109.

36. XM Leverve et al. Mitochondrial metabolism and type-2 diabetes: a specific target of metformin. Diabetes Metab. 2003;29:6s88-94.

37. Liu X, Romero IL, Litchfield LM, Lengyel E, Locasale JW. Metformin Targets Central Carbon Metabolism and Reveals Mitochondrial Requirements in Human Cancers. Cell Metab. 2016;24:72839.

38. Finley J. Cellular stress and AMPK activation as a common mechanism of action linking the effects of metformin and diverse compounds that alleviate accelerated aging defects in Hutchinson-Gilford progeria syndrome. Med Hypotheses. 2018;118:151-62.

39. J Li et al., Metformin limits osteoarthritis development and progression through activation of AMPK signalling. Ann Rheum Dis, (2020).

40. Liu M, Zhang Z, Wang H, Chen X, Jin C. Activation of AMPK by metformin promotes renal cancer cell proliferation under glucose deprivation through its interaction with PKM2. Int J Biol Sci. 2019;15:617-27.

41. Szrejder M, et al. Metformin reduces TRPC6 expression through AMPK activation and modulates cytoskeleton dynamics in podocytes under diabetic conditions. Biochim Biophys Acta Mol Basis Dis. 2020;1866:165610.

42. Y Wang et al. Metformin Improves Mitochondrial Respiratory Activity through Activation of AMPK. Cell Rep. 2019;29:1511-23.e1515.

43. D Benjamin et al. Dual Inhibition of the Lactate Transporters MCT1 and MCT4 Is Synthetic Lethal with Metformin due to NAD + Depletion in Cancer Cells. Cell Rep. 2018;25:3047-58.e3044.

44. Blumrich EM, Dringen R. Metformin Accelerates Glycolytic Lactate Production in Cultured Primary Cerebellar Granule Neurons. Neurochem Res. 2019;44:188-99.

45. Huang W, Castelino RL, Peterson GM. Lactate Levels with Chronic Metformin Use: A Narrative Review. Clin Drug Investig. 2017;37:991-1007.

46. Piel S, Ehinger JK, Elmér E, Hansson MJ. Metformin induces lactate production in peripheral blood mononuclear cells and platelets through specific mitochondrial complex I inhibition. Acta Physiol (Oxf). 2015;213:171-80.

47. RL Wolfson et al. Sestrin2 is a leucine sensor for the mTORC1 pathway. Science. 2016;351:43-8.

48. Dodd KM, Tee AR. Leucine and mTORC1: a complex relationship. American Journal of PhysiologyEndocrinology Metabolism. 2012;302:E1329-42. 
49. Kimball SR, Gordon BS, Moyer JE, Dennis MD, Jefferson LS. Leucine induced dephosphorylation of Sestrin2 promotes mTORC1 activation. Cell Signal. 2016;28:896-906.

50. R Zoncu et al. mTORC1 senses lysosomal amino acids through an inside-out mechanism that requires the vacuolar H(+)-ATPase. Science. 2011;334:678-83.

51. L Chantranupong et al. The CASTOR Proteins Are Arginine Sensors for the mTORC1 Pathway. Cell. 2016;165:153-64.

52. Folch J, Ascoli I, Lees M, Meath JA, Le BN. Preparation of lipide extracts from brain tissue. J Biol Chem. 1951;191:833-41.

53. AP Coll et al. GDF15 mediates the effects of metformin on body weight and energy balance. Nature. 2020;578:444-8.

54. Hamed HO. Role of adiponectin and its receptor in prediction of reproductive outcome of metformin treatment in patients with polycystic ovarian syndrome. J Obstet Gynaecol Res. 2013;39:1596-603.

55. Stern JH, Rutkowski JM, Scherer PE. Adiponectin, Leptin, and Fatty Acids in the Maintenance of Metabolic Homeostasis through Adipose Tissue Crosstalk. Cell Metab. 2016;23:770-84.

56. JR Su et al. Relationship of Serum Adiponectin Levels and Metformin Therapy in Patients with Type 2 Diabetes. Horm Metab Res. 2016;48:92-8.

57. MA Alfaqih et al. Serum Branched Chain Amino Acids Are Associated with Type 2 Diabetes Mellitus in Jordan. Korean J Fam Med. 2018;39:313-7.

58. JL Flores-Guerrero et al., Plasma Branched-Chain Amino Acids and Risk of Incident Type 2 Diabetes: Results from the PREVEND Prospective Cohort Study. J Clin Med 7, (2018).

59. Ruiz-Canela $M$, et al. Plasma branched chain/aromatic amino acids, enriched Mediterranean diet and risk of type 2 diabetes: case-cohort study within the PREDIMED Trial. Diabetologia. 2018;61:156071.

60. Zhao L, et al. Association of Circulating Branched-Chain Amino Acids with Gestational Diabetes Mellitus: A Meta-Analysis. Int J Endocrinol Metab. 2019;17:e85413.

61. Thomas CR, Turner SL, Jefferson WH. C. J. Bailey, Prevention of dexamethasone-induced insulin resistance by metformin. Biochem Pharmacol. 1998;56:1145-50.

62. Bligh EG, Dyer WJ. A rapid method of total lipid extraction and purification. Can J Biochem Physiol. 1959;37:911-7.

\section{Supplemental Figure Legend}

\section{Supplementary Figure 1. Late application of metformin-diet fails on expanding lifespan of iMSUD Mice.}
A) Survival assay of male and female iMSUD mice fed with metformin diet ( $0.1 \%$ in 5 LG6 diet, Newco Distributor) started at 21 days-old age. iMSUD mice with control diet (iMSUD_control diet, $n=21$ ); iMSUD mice with metformin diet (iMSUD_metformin diet, $n=28$ ). 
B) Body weight recording of metformin diet treated wild type and iMSUD mice. Wild type mice with control diet (WT_control diet, $n=30$ ); wild type mice with metformin diet (WT_metformin diet, $n=45$ ); iMSUD mice with control diet (iMSUD_control diet, $n=21$ ); iMSUD mice with metformin diet (iMSUD_metformin diet, $n=28$ ).

\section{Supplementary Figure 2. Metformin-induced increased activation of IGF-1R signals in iMSUD Mice.}

A) Western blots of phospho-IGF-1R and IGF-1R in liver and skeletal muscle of wild type and iMSUD mice treated with PBS or metformin. Adjoining bar graph shows quantitation.

B) Metformin has minor effect on glucose tolerance in wild type mice. Glucose tolerance assay were performed on at 50 days-old wild type animals with administration of glucose IP (1.2 g/ kg BW). Wild type with PBS treated for 50day (WT_PBS_50 days, $n=2)$; wild type mice with metformin $(250 \mathrm{mg} / \mathrm{kg} \mathrm{bw})$ treated for 50 days (WT_MET_50 days, $n=21$ ). AUC, area under curve. Data are mean \pm s.e.m. $P$ value by t-test.

\section{Supplementary Figure 3. Effect of metformin on TCA metabolites in liver samples.}

Heat map of carbohydrate, nucleotide and amino acid metabolism in wild type and iMSUD liver samples. The colors show relative comparative level to wild type PBS sample in different group. Data are shown as mean \pm s.e.m. ( $n \geq 3$ per group).

\section{Supplementary Figure 4. Effect of metformin on TCA metabolites in skeletal muscle samples.}

Heat map of carbohydrate, nucleotide and amino acid metabolism in wild type and iMSUD skeletal muscle samples. The colors show relative comparative level to wild type PBS sample in different group. Data are shown as mean \pm s.e.m. ( $n \geq 3$ per group).

\section{Figures}


A

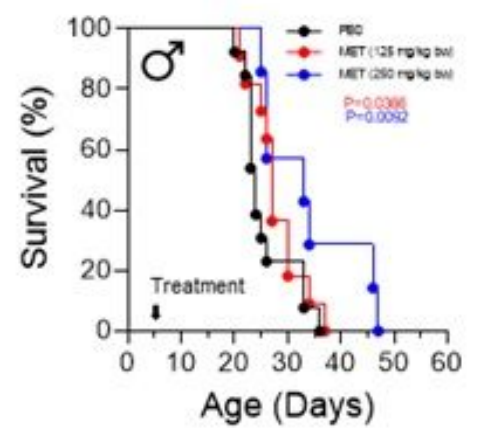

C

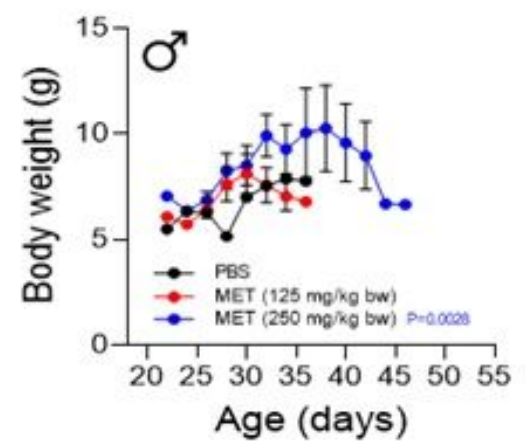

$\mathrm{E}$

G
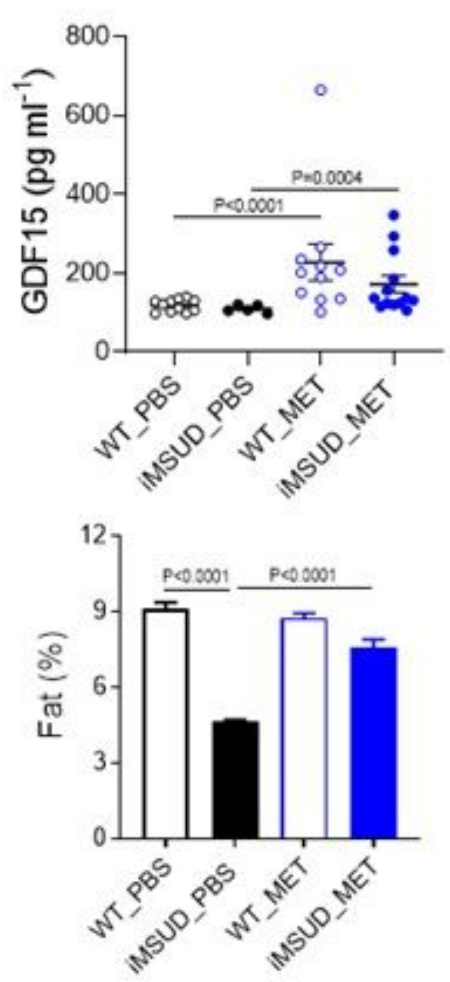

B
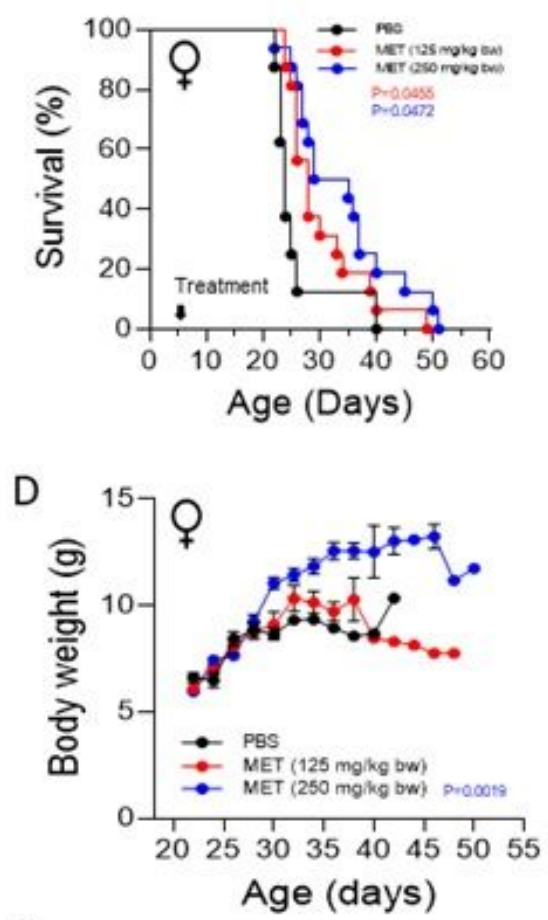

$\mathrm{F}$

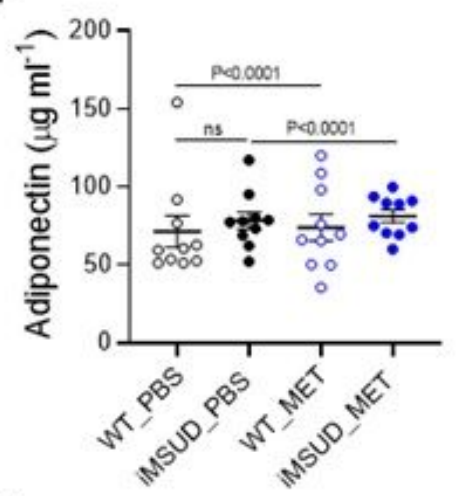

$\mathrm{H}$

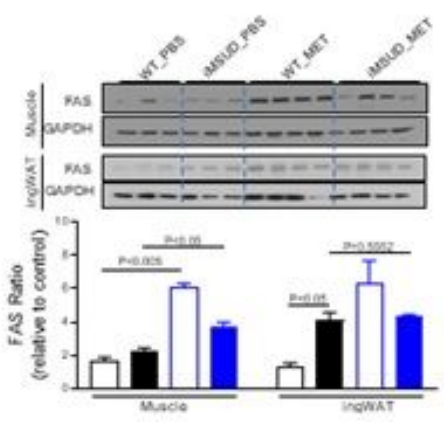

\section{Figure 1}

Metformin improves overall survival, and body fat composition in iMSUD mice. A\&B) Survival of iMSUD mice administrated metformin (MET, 125 or $250 \mathrm{mg} / \mathrm{kg} \mathrm{bw}$ ) by daily intraperitoneal injection started from 5 days of age. ( $n \geq 7$ per group). Survival curves were analyzed with the log-rank test. C\&D) Body weight (BW) recording of iMSUD mice. Data are show as mean \pm s.e.m ( $n \geq 7$ per group). E) Serum GDF15 level in iMSUD mice measured 3-4 weeks after daily metformin IP (250 mg/kg bw). Data are shown using mean 
\pm s.e.m ( $n \geq 5$ /group); P by one-way ANOVA assay and t-test. WT: wild type. F) Serum adiponectin concentration. Data are shown using mean \pm s.e.m ( $n \geq 10$ /group); P by one-way ANOVA assay and t-test. WT: wild type. G) Measurement of total fat content in iMSUD mice treated with metformin at 22 days age. H) Elevated protein expression of fatty acid synthase (FAS) in the skeletal muscle and subcutaneous fat tissue in metformin-treated iMSUD mice. Muscle: gastrastrocnemius muscle; ingWAT: subcutaneous fat. Quantitation of blots are displayed using mean \pm s.e.m in the bar-graphs.

A

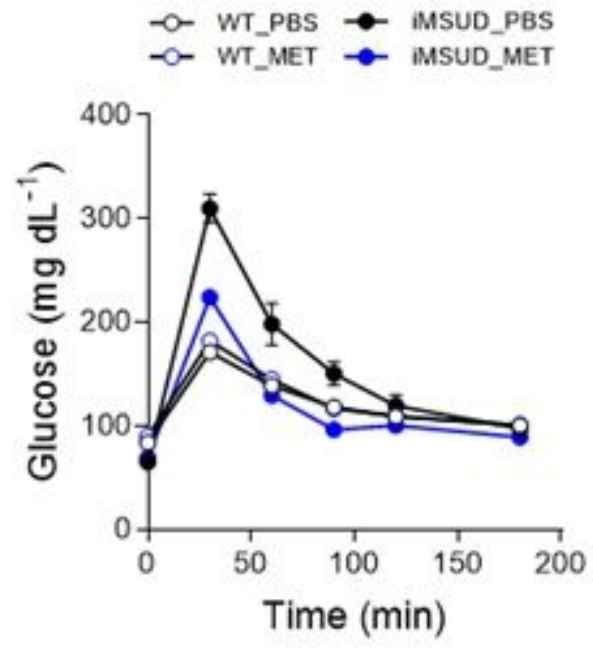

C

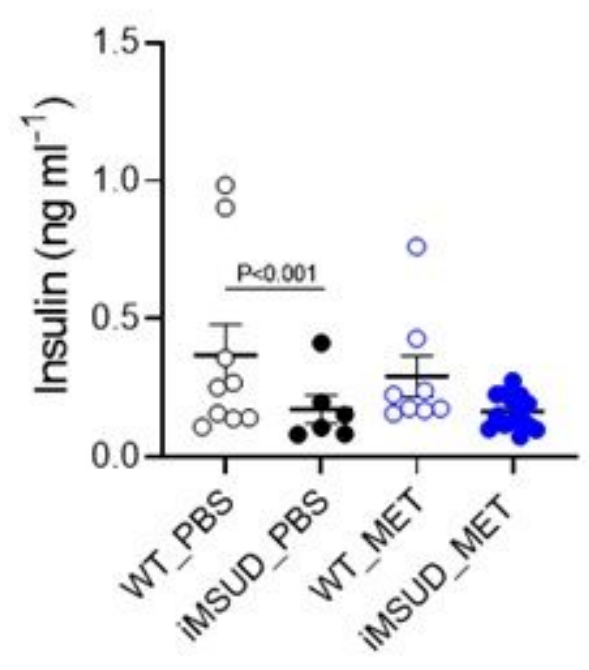

B

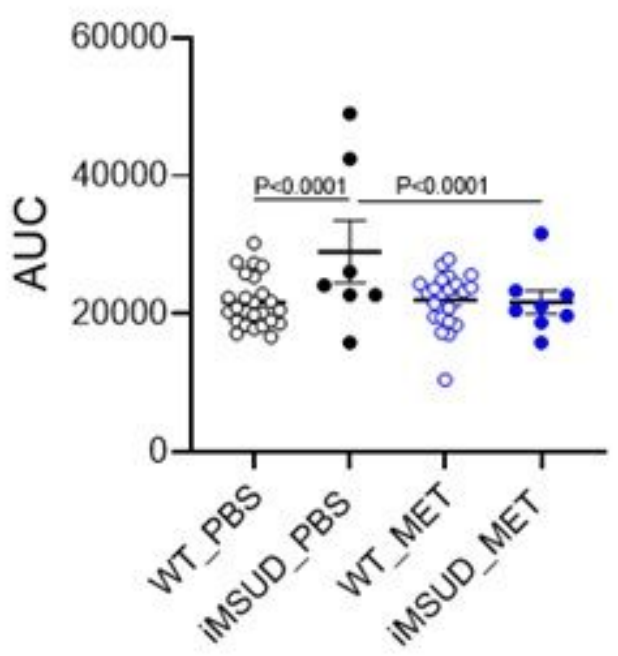

D

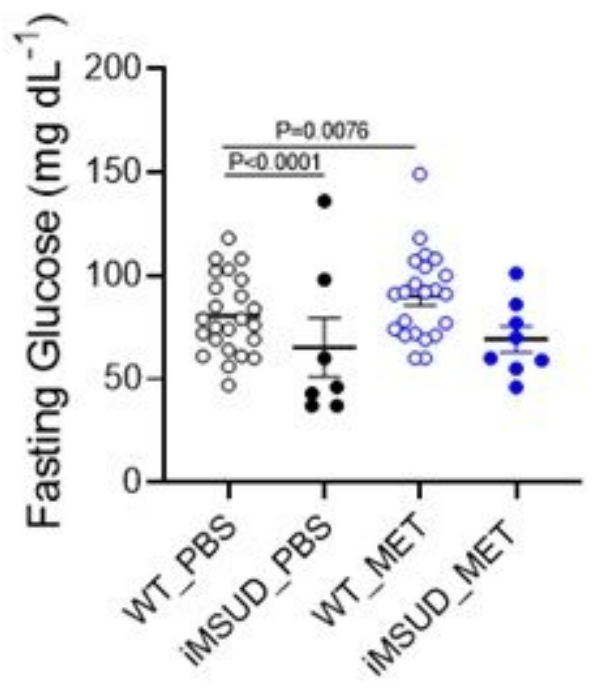

Figure 2

Effect of metformin on glucose homeostasis, the level of insulin and fasting glucose in iMSUD mice. A\&B) Metformin improves glucose tolerance in iMSUD mice. Glucose tolerance assessed were perform on at 27 days-old animals with administration of glucose IP (1.2 g/ kg BW). Wild type mice with PBS 
treatment (WT_PBS, $n=26$ ); wild type mice with metformin treatment (WT_MET, $n=22)$; iMSUD mice with PBS treatment (iMSUD_PBS, $n=7$ ); iMSUD mice with metformin treatment (iMSUD_MET, $n=8$ ). AUC, area under curve. Data are mean \pm s.e.m. P value by one-way ANOVA. C) Serum insulin in metformintreated wilt type and iMSUD mice. Wild type mice with PBS treatment (WT_PBS, $n=9$ ); wild type mice with metformin treatment (WT_MET, $n=8$ ); iMSUD mice with PBS treatment (iMSUD_PBS, $n=6$ ); iMSUD mice with metformin treatment (iMSUD_MET, $n=11$ ). Data are mean \pm s.e.m. $P$ value by one-way ANOVA. D) Fasting glucose concentration in mice treated as A\&B. Data are mean \pm s.e.m. P value by one-way ANOVA. 
A

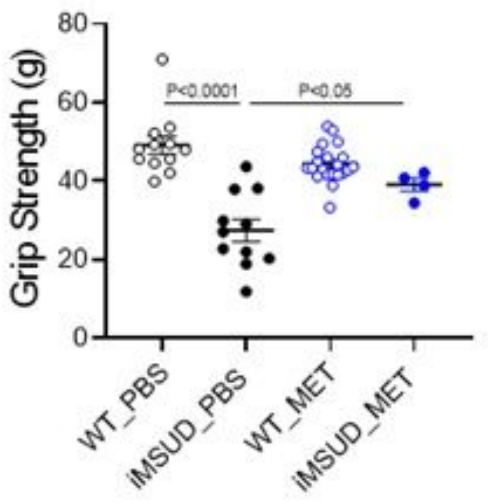

B
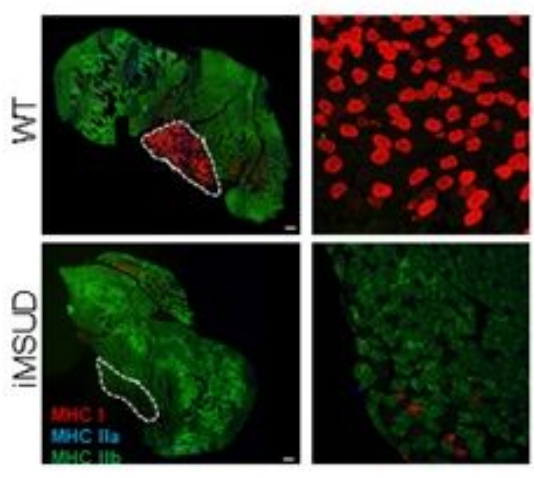

C

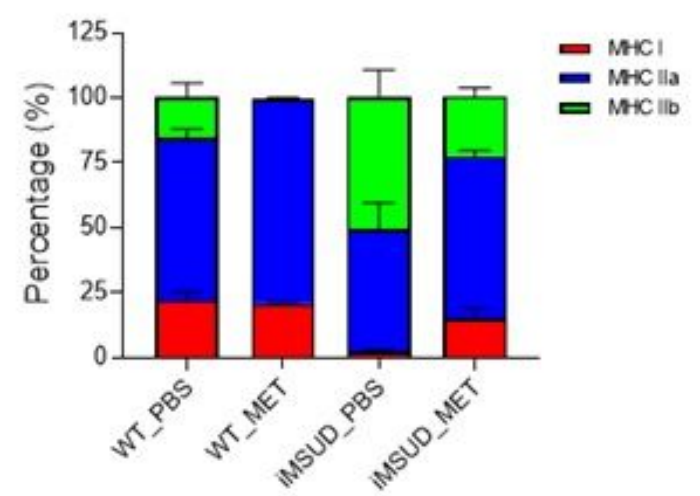

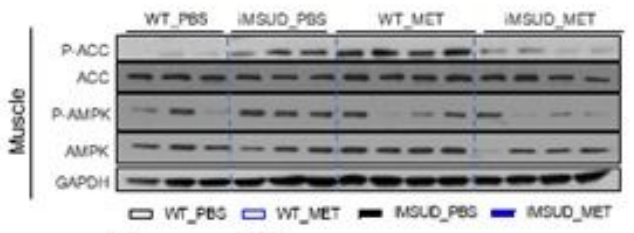
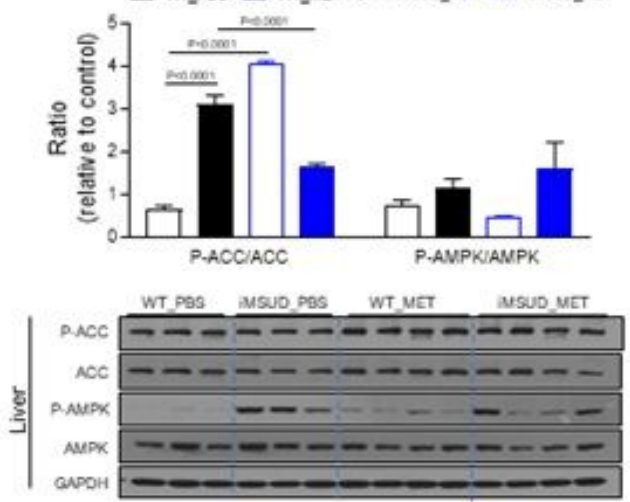

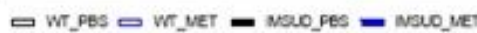

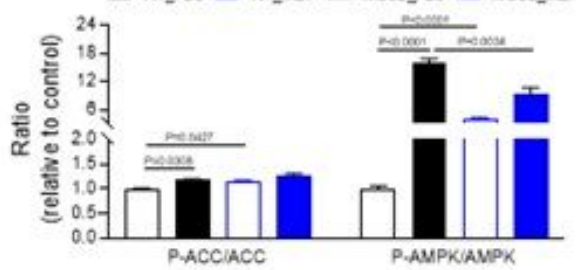

\section{Figure 3}

Partial restoration of skeletal muscle fiber type and physiological function by metformin. A) Physiological fore limb grip strength assay in metformin-treated iMSUD mice. The skeletal muscle physiological function was assayed by grip strength meter. Wild type mice with PBS treatment (WT_PBS, $n=12$ ); wild type mice with metformin treatment (WT_MET, $n=22$ ); iMSUD mice with PBS treatment (iMSUD_PBS, $n=$ 11); iMSUD mice with metformin treatment (iMSUD_MET, $n=4$ ). Data are shown as mean \pm s.e.m. $P$ 
value by one-way ANOVA and t-test. B) Distribution of muscle fiber types in skeletal muscle samples of metformin-treated iMSUD mice. The muscle samples were stained with different muscle fiber type markers (MHC type I, red; MHC type lla, blue; and MHC type llb, green). The images were captured using confocal microscope (Zeiss LSM780) and recomposed using ImageJ software. Scale bar: $50 \mu \mathrm{m}$. C) Quantification of muscle fiber type distribution in soleus muscle. Data are shown as mean \pm s.e.m. $(\mathrm{n}=$ 3/group). D) Quantification of AMPK and ACC activation in skeletal muscle and liver samples. Data are shown as mean \pm s.e.m. ( $n=3$ or 4 /group). $P$ value by one-way ANOVA and t-test.

A
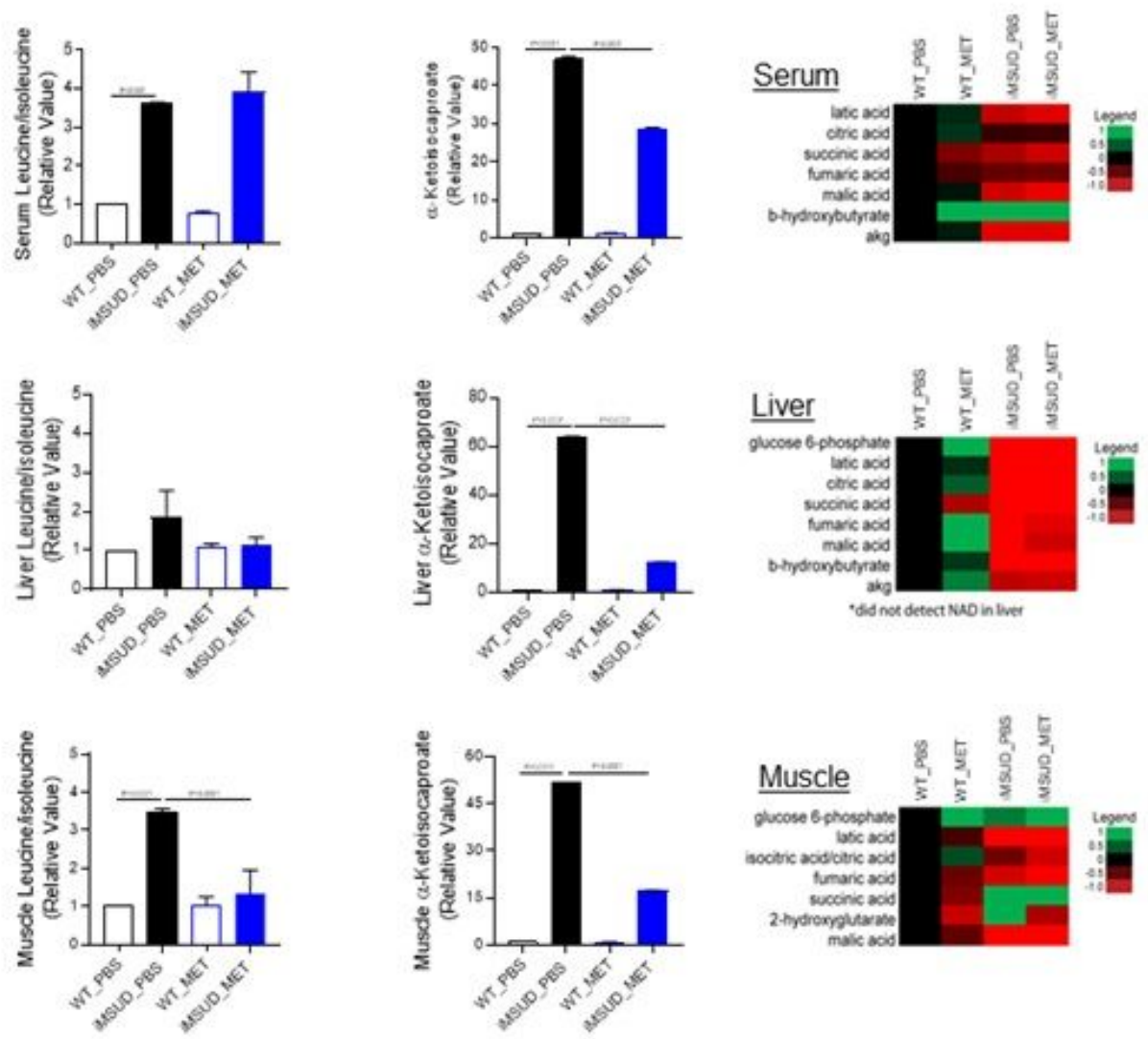

B

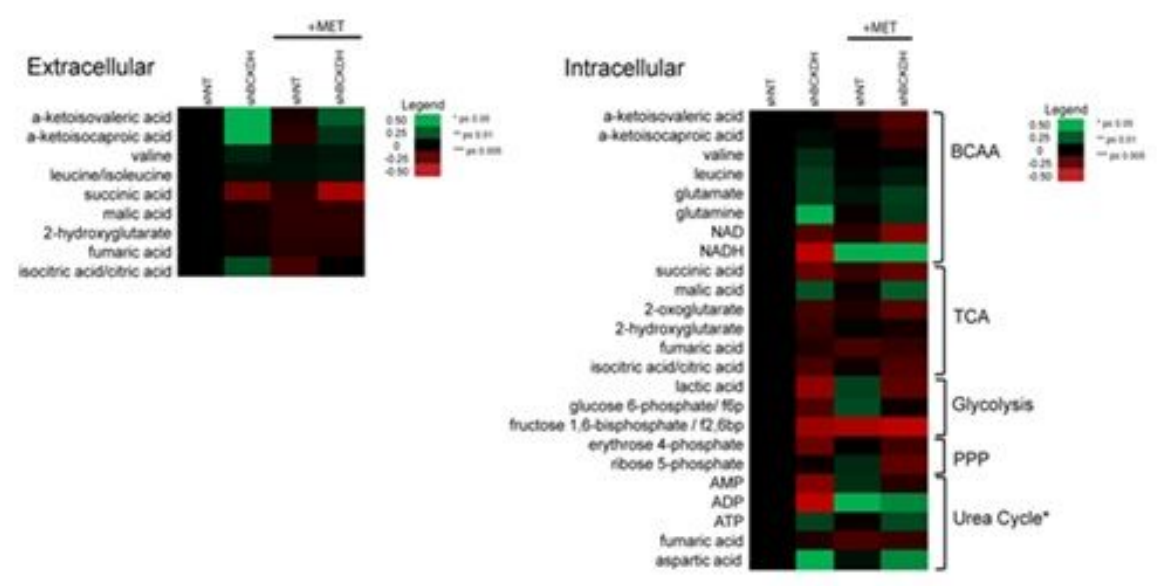




\section{Figure 4}

Partial restoration of TCA metabolites by metformin. A) Heat map of glycolytic and TCA metabolites. The relative level of glycolytic and TCA metabolites from liver, skeletal muscle (gastrastrocnemius) and serum of animals were determined by LC-Mass assay. The bar graphs show relative levels of leucine/isoleucine and ketoisocaproic acid (KIC) in different group ( $n=3$ or 4/group). The data was shown as mean \pm s.e.m. B) Heat map shows relative levels of metabolites from $\mathrm{C} 2 \mathrm{C} 12$ cells (extracellular and intracellular portion) with shNT, shBCKDH with or without metformin (MET) treatment.

A

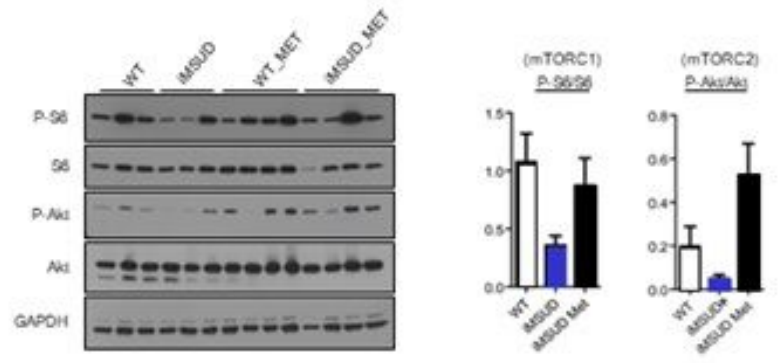

B
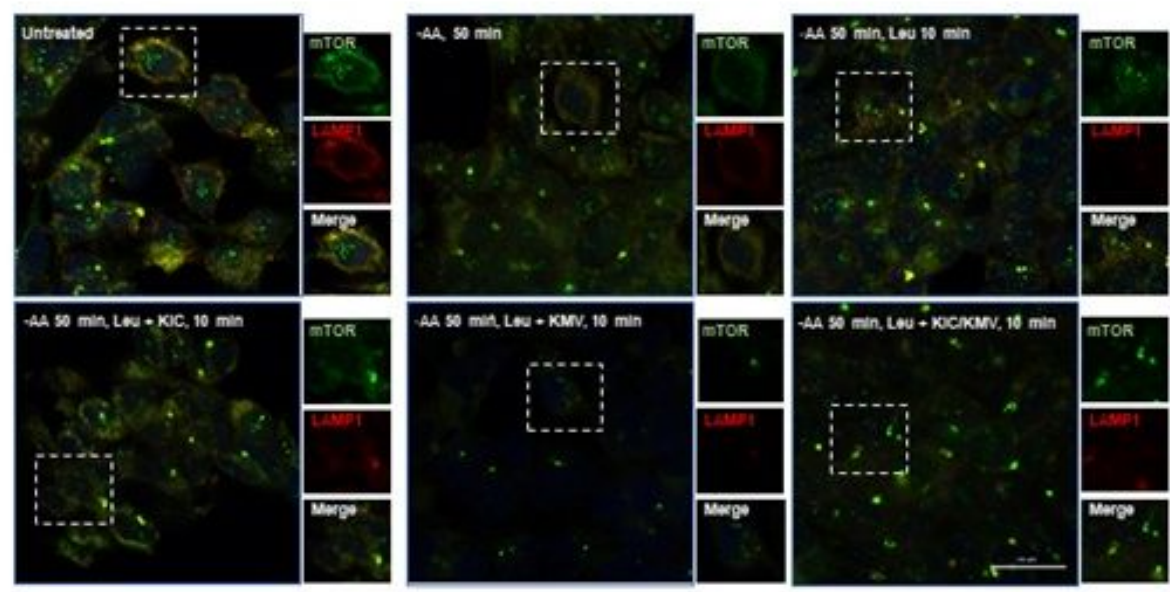

C

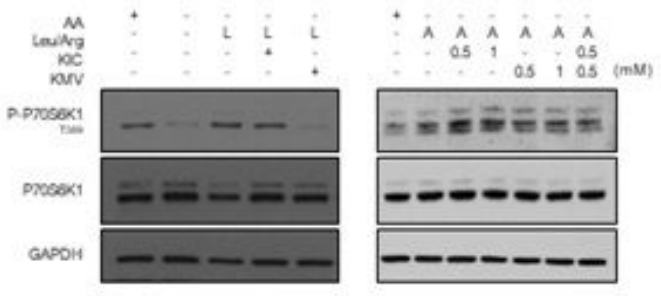

D

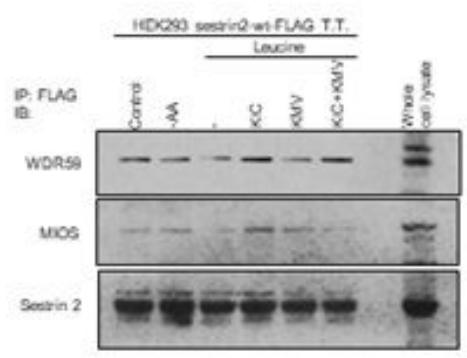

E
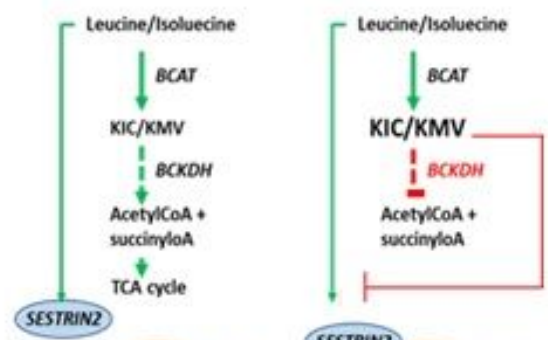

SESTRIN2 GATOR2 GATOR1

1

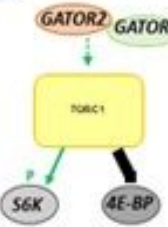

566 


\section{Figure 5}

Inhibition of leucine mediated activation of mTOR in iMSUD A) Restoration of mTORC1 (p-S6) and mTORC2 (p-Akt) signaling by metformin treatment in iMSUD mice in skeletal muscle (gastrastrocnemius). B) Ketoisocaproic acid (KIC) and ketomethylvaleric acid (KMV) inhibit leucineinduced lysosomal translocation of mTOR in HEK293T cell. Lysosomal marker LAMP1-red; mTOR-green; co-localization- yellow. Scale bar: $20 \mathrm{Im}$. C) Inhibition of leucine-mediated, but not arginine-mediated mTORC1 activation (as judged by levels of p-P70S6K) by KIC (1mM) and KMV (1mM) in HEK293T cells. D) Inhibition of sestrin2-GATOR2 binding by KIC (1 mM) and KMV (1 mM) treatment in HEK293T cells measured by immunoprecipitation of FLAG-sestrin2, and probing with antibodies against GATOR2 components, WDR59 and MIOS. E) Cartoon depicting model of KIC/KMV mediated inhibition of leucine mediated mTOR activation in iMSUD mice.

\section{Supplementary Files}

This is a list of supplementary files associated with this preprint. Click to download.

- SIFig1.jpg

- SIFig2.jpg

- SIFig3.jpg

- SIFig4.jpg 